NASA Technical Memorandum 84600

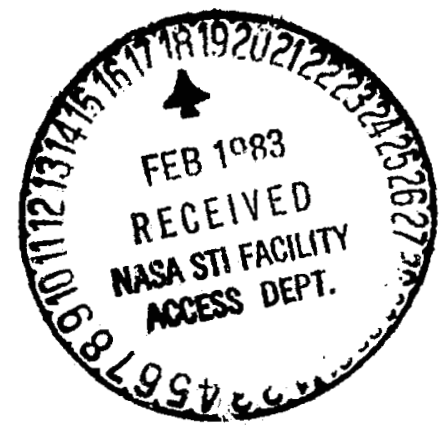

SUPERSONIC AERODYNAMIC CHARACTERISTICS OF A TAIL-CONTROL CRUCIFORM MANEUVERABLE MISSILE WITH AND WITHOUT WINGS

(NASA-TM-84600) SUPERSONIC AEFODYNAMIC
SAARACTERISTICS OF 1 TAIL-CCNTECL CEUCIFOEM
MANEUV ERABLE MISSILE NITH AND WITHCUT WINGS

(NASA) 13 P HC A02/UF AOI CSCL 0 IA $\begin{array}{ll}\text { Unclas } \\ \text { G3/02 } & 02752\end{array}$

\title{
M. Leroy Spearman
}

January 1983

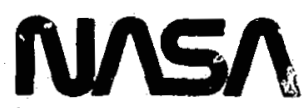

National Aeronautics and

Space Administration 


\section{SUMMARY}

The aerodynamic characteristics for a winged and a wingless cruciform missile configuration were examined. The configuration had an ogive-cylinder body with a 3.5 caliber forebody; an overall length-to-diameter ratio of 11.667; and had cruciform tails that were trapezoidal in planform. Tests were made both aith and without 72.9-degree cruciform delta wings. The investigation was made for Mach numbers from 1.50 to 4.63 , roll attitudes of 0 degrees and 45 degrees, angles of attack from -4 degress to 22 degrees, and tail control deflections from 10 degrees to -40 degrees.

In general, the results indicated that the winged missile with its more linear aerodynamic characteristics and higher lift-curve slope, should provide the highest maneuverability over a large operational range. The wingless missile, with a lower lift-curve slope and more nonlinear characteristics, but with lower minimum drag, might be more suitable for missions where acceleration time is important and where 1 ift can be generated from high dynamic pressure incurred at low altitudes or at higher Mach numbers. Hence, final trades in missile/mission concepts may lead to two extremes--a missile capable of reaching the target area rapidly but being unable to perform the required end-game maneuver, or, a missile that has the potential of nerforming a highly maneuverable intercept but may have limitations in the time requirad to reach the target area. A suitable concept between the two extremes may be difficult to achieve if a single system is required to operate over a wide range. A preferred solution may be to design specific missile configurations to perform specific mission requirements, thus giving the user a wider choice of weapons to fill a variety of possible mission needs.

\section{INTRODUCTION}

A continual need exists for reviewing and updating the state-of-the-art in maneuverable missile concerts. Of cirrent interest are means for improving missile effectiveness while at the same time reducing the complexity and cost, particularly for highly maneuverable air-to-air or surface-to-air missiles. Factors that affect missile effectiveness include aerodynamics, guidance and control, propulsion, structures, and warhead and fusing. Complete trade studies, of course, must consider such things as size, weight, carriage and storage, launcher compatibility, and system complexity. Each of these factors can be impacted by the configuration aerodynamics, however. Therefore, it was the purpose of this study to examine the aerodynamic behavior of a cruciform, aft-tail control missile both with and without a highly swept delta wing in order to assess the relative merits of the concepts and to consider possible implications on design choice. Basic data for the concepts are presented in references 1 and 2 . There being no unusually significant characteristic difference between the results obtained at roll attitudes of 0 degrees and 45 degrees, only illustrative results for a roll attitude of 45 degrees are used in the present study. 


\section{SYMBOLS}

The results are referred to the stability axis system. The coeffictents, symbols, and abbreviations are defined as follows:

A maximum cross-sectional area of bods

a.c. aerodynamic center

an instantaneous normal acceleration in g-units

$\mathrm{C}_{\mathbf{D}}$

drag coefficient, $\frac{\text { drag }}{q A}$

$C_{0,0} \quad$ drag coefficient at $\alpha=0^{\circ}$

c.g. center of gravity

$C_{L}$

lift coefficient, $\frac{\text { lift }}{q A}$

$C_{L_{\alpha}}$

lift-curve slope at $\alpha=0^{\circ}, \frac{\partial C_{L}}{\partial \alpha}$

$\mathbf{C}_{\mathbf{m}}$

pitching-moment coefficient, $\frac{\text { pitching moment }}{q A_{l}}$

h

a) titude

body length

Mach number

$q$

dynamic pressure

$W$

weight

$x_{a c}$

location of aerodynamic center, percent body length

$\alpha$ angle of attack, degrees

$\delta$

pitch-control deflection, degrees, positive trailing edge down

$\phi$

roll angle of wing-chord plane with respect to lateral reference plane, degrees 


\section{APPARATUS}

\section{Tunnels}

The investigation was conducted in the Langley Unitary plan wind tunnel which is a variable-pressure continuous-flow facility. The Unitary plan wind tunnel has two 1.22-meter-square test sections and the nozzles leading to the test sections are of the asymmetric sliding-block type which permits a continuous variation in test-section Mach numbers from 1.5 to 2.9 in the low Mach number test section and from 2.3 to 4.7 in the high Mach number test section.

\section{Mode1}

Dimensional details of the model are shown in figure 1 . The body was a cylinder with a 3.5-caliber forebody and an overall length-diameter ratio of 11.667 . The wings and tails were made of flat plates with approximately a 21.5 degree angle normal to the leading and trailing edges. The wings had a leading-edge sweep angle of 72.9 degrees, an exposed panel aspect ratio of 1.23 , a root thickness ratio of about 1.5 percent, and a ratio of total span to body diameter of 3.667. The tails had a trapezoidal planform symmetrical about a 0.50 -chord hinge line and were made from flat plates having an average thickness ratio of about 8.4 percent. Tail deflection angles of $0,10,20,30$, and 40 degrees were provided.

\section{Tests}

Tests were made for Mach numbers of $1.50,1.90,2.36,2.86,3.95$ and 4.63. The Reynolds number was $8.2 \times 10^{6}$ per meter. The dewpoint was maintained sufficiently low to assure negligible condensation effects. In order to provide boundary-layer transition to turbulent conditions, $0.16-\mathrm{cm}$ wide strips of No. 60 carborundum grit were placed $1.02 \mathrm{~cm}$ aft of the leading edge of the wings (measured streamwise), just behind the leading-edge bevel on the tails, and $2.54 \mathrm{~cm}$ aft on the model nose.

The angle of attack was varied from about -4 degrees to 22 degrees. Results were obtained with the surfaces horizontal and vertical $\left(\phi=0^{\circ}\right)$ and with the surfaces in 45 -degree planes $\left(\phi=45^{\circ}\right)$. Illustrative results at $\phi=45^{\circ}$ only are used in the present paper. Aerodynamic forces and moments on the model were measured by means of a six-component electrical strain-gage balance which was housed within the model.

The angle of attack has been corrected for deflection of the balance and sting due to aerodynamic loads; angles of attack have also been corrected for tunnel airflow misalignment. The results have been adjusted to correspond to freestream static pressure acting over the model base. Values for the base axial-force coefficients can be found in references 1 and 2 .

Detailed results for both the 0 -degree and the 45-degree roll $\mathrm{planes}$ may be found in references 1 and 2. In addition, roll-control data for differential deflection of the tails will be found for the winged model in reference 1 . 


\section{DISCUSSION}

\section{Longitudinal Aerodynamic Characteristics}

Representative longitudinal results for both the winged and the wingless missiles are presented in figures 2 to 4 for Mach numbers of 1.90 and 4.63 for $\phi=45^{\circ}$ and a reference center of gravity of 60 percent $\mathrm{l}$. The lift and drag characteristics at $M=1.90$ for $\delta=0^{\circ}$ and $-20^{\circ}$ (fig. 2) indicate that the winged missile provides substantially higher lift for a given angle of aitack or will maintain a given lift at substantially lower angles of attack. A desirable goal for a maneuvering missile would obviously be to attain the highesc lift possible at the lowest angle possible. The lift available is directly related to the maneuverability. The angle of attack has several implications related to regions at which flow separation (and possible aerodynamic nonlinearities) occur, inlet flow characteristics for airbreathing propulsion systems, and "look-angle" requirements for guidance seekers. The slightly more nonlinear variation of $C_{L}$ with $\alpha$ for the wingless missile results from, the influence of the forebody lift which increases with increasing $\alpha$. This effect is less evident with the winged missile since the total lift is dominated more by the wing lift than the forebody ifft.

The drag-due-to-ifft is less for the winged missile thus offering the possibility of higher usable lift and greater maneuverability. At the higher lifts obtainable with the winged missile, the maximum values of total drag are, of course, substantially higher than those incurred with the wingless missile. An obvious trade to consider here is the amount of lift (or maneuverability) required and the amount of thrust (or speed) imposed. As an indication of the thrust requirements, a drag coefficient of 4 equates to about 3,400 pounds for an 8-inch diameter missile at 20,000 feet and $M=1,90$.

The trim, stability, and control characteristics at $M=1.90$ (fig. 3 ) indicate a more linear variation of $C_{m}$ with $C_{L}$ and higher values of trim $C_{L}$ for the winged missile. The nonlinear variation of $C_{m}$ with $C_{L}$ for the wingless missile reflects the dominate influence of the forebody lift which increases with $\alpha$ and results in a forward shift of center of pressure. Because of the decrease in stability and the slightly higher control effectiveness, it would be possible to trim the wingless missile at the lower lifts with less control deflection and less trim drag than would be required for the winged missile. Here again the trade effects involve the lower thrust requirements for the wingless missile at the expense of higher lift capability.

Similar aerodynamic results for the winged and the wingless missile at $M=4.63$ are shown in figure 4 . The lift, drag, and trim characteristics are essentially the same as those at $M=1.90$. However, the increased control effectiveness for the wingless missile is somewhat more pronounced and the decrease in stability is more severe for the case of a constant c.g. location. In fact, for this Mach number, a forward shift in c.g. would be required in order to provide static longitudinal stability and trim for the wingless missile.

It is recognized that weight differences due to wing structure is also a necessary consideration to a comparative study. However, it is believed that for a maneuvering missile, the aerodynamic differences would be of more significance than wing weight differences. 


\section{Longitudinal Aerodynamic Parameters}

Three key longitudinal parameters--the lift curve slope, the minimum drag, and the aerodynamic center location--are summarized as a function of Mach number in figure 5 for the two missiles. The substantially higher $C_{L_{\alpha}}$ for the winged missile and the higher $C_{D, 0}$ are evident across the Mach number range. The lift-curve slope is increased by a factor of 2 to 3 and has important implications on weight-sustaining capability as well as on maneuverability. The minimum drag difference, while seemingly small, could have important implications on the thrust requirements and the fly-out time during flight under nearly ballistic (zero lift) conditions. It should be remembered, however, that the lower drag-due-to-lift for the winged missile tends to offset the minimum drag penalty as the flight lift coefficient increases.

A considerably greater variation in aerodynamic center location with $M$ occurs for the wingless missile than for the winged missile. This variation is caused by the changes in forebody lift, which is a primary producer of lift for the wingless missile, and results in complicating the problem of maintaining a compatible c.g./a.c. location for static stability over the Mach number range. The wingless missile was found to be stable at Mach numbers from about 1.5 to 2 with the c.g. as far aft as 70 percent 2. However, in order to provide static stability for Mach numbers up to about 4.7, the c.g. for the wingless missile would have to be at about 50 percent with the probability then of excessively high stability levels at lower Mach numbers. The small variation of $x_{a c}$ for the winged missile (about 5 percent 2 ) considerably relieves the problem of c.g./a.c. compatibility. With the c.g. at 60 percent $l$, for example, the winged missile is stable over the $M$ range from 1.50 to 4.63 whereas the wingless missile is unstable above about $M=3$.

\section{Maneuver Characteristics}

The tail trim lift effectiveness for the winged missile at various c.g. locations is shown in figure 6 for all test Mach numbers at $\phi=45^{\circ}$ and for tail deflections to -40 degrees. These results show a general decrease in effectiveness with increasing $\delta$ at the lower Mach numbers for the more forward c.g.'s. It is important to notice that this characteristic changes with increasing $M$, however, and the effectiveness increases progressively up to the maximum deflection angle. This characteristic is indicative of the beneficial effects of the local increase in dynamic pressure in the vicinity of the tail and is apparent either with or without the wing. The results of figure 6 also show the expected increase in trim capability as the c.g. moves rearward. The results indicated that a c.g. location of 62 percent $z$ was the most rearward for which positive static stability could be maintained for the winged missile throughout the range of these tests. Similar trim lift curves were developed for the wingless missile and indicated that the most rearward permissible c.g. over the range of these tests was about 51 percent 2 . The trim lift attainable, as depicted by such curves, when divided by the lift required for a specified weight and altitude, determine the instantaneous normal acceleration potentially available.

An illustration of the normal acceleration potentia? for a $\mathrm{W} / \mathrm{A}$ of $35.9^{\circ} \mathrm{kN} / \mathrm{m}^{2}$ with the c.g. at 60 percent $l$ is shown in figure 7 for the winged and wingless missiles at three altitudes. These curves are arbitrarily limited to a maximum $g$ of 50 or to the Mach number at which static instability occurred. Operational bounds for given flight conditions can be determined from such curves and similar curves can 
be developed for other altitudes, loading conditions, or c.g. 1ocations. Several observations can be made from the results shown in figure 7 . For example, the $a_{n}$ values for a given flight condition are always less for the wingless missile than for the winged missile as would be expected from the difference in lift capability. At the lower altitudes, the winged missile indicated g-loadings at Mach numbers above about 3 that would probably approach normal structural limitations. In addition, significant high altitude $g$ capability is indicated for the winged missile, especially when flying at the higher Mach numbers. For example, for the highest altitude shown $(21,336 \mathrm{~m}$ or $70,000 \mathrm{ft})$, the winged missile at $M=4.63$ has an $a_{n}$ value of about $18 \mathrm{~g}^{\prime} \mathrm{s}$.

There are regions where substantial $g$ capability exists for the wingless missile. At $h=6,096 \mathrm{~m}(20,000 \mathrm{ft})$, for example, the wingless missile achieves an $a_{n}$ of about 26 at $M=2.86$. The same $g$-level for the winged missile occurs at a somewhat lower Mach number of about 1.8. Hence, the advantages of speed and lower a)titudes for developing dynamic pressure are apparent. Higher speed for a constant g-level also results in a larger turning radius, however. A definite advantage of the higher possible speed for the wingless missile would be in the reduced fly-out time during acceleration toward the target before end-game maneuvering is required. This time to intercept can be extremely important.

\section{CONCLUDING REMARKS}

A study has been made of the aerodynamic characteristics of a tail-control cruciform maneuverable missile with and without 72.9 degrees cruciform delta wings at Mach numbers from 1.50 to 4.63 .

The results indicated that the winged missile provides substantialiy higher lift for a given angle of attack or will provide a given lift at substantially lower angles of attack. The winged missile does, of course, have higher values of drag near zero $1 \mathrm{ift}$ but the drag-due-to-lift is lower and for maneuvering filight tends to offset the minimum drag penalty. The winged missile has more linear pitching moment characteristics and substantially less aerodynamic center shift with Mach number. The presence of the wing does slightly reduce the control effectiveness of the tail. However, the winged missile, for a given flight condition, always provides the highest potential instantaneous normal acceleration.

The wingless missile should be capable of higher longitudinal acceleration during ballastic flight because of lower minimum drag. Hence, final trades in missile/mission concepts may evolve to two possible extremes--a missile capable of reaching the target area rapidly but being unable to perform the required end-game maneuver, or a missile that has the potential of performing a highly maneuverable intercept but may have limitations in the time required to reach the target area. The best possible concept lies somewhere between the two extremes and, even with careful trade studies, would be difficult to achieve if a single system is required to operate over a wide range. An obvious preferred solution would be to design specific missile configurations to perform specific mission requirements, thus giving the user a wider choice of weapons to fill a variety of possible mission needs. 


\section{REFERENCES}

1. Spearman, M. Leroy; and Fournier, Roger H.: Aerodynamic Characteristics of a Maneuverable Missile With Cruciform Delta Wings and Aft Tail Controls at Mach Numbers From 1.50 to 4.63. NASA TM X-1863, 1969.

2. Spearman, M. Leroy; and Fournier, Roger H.: Aerodynamic Characteristics of a Wingless Maneuverable Missile With Cruciform Aft Tail Controls at Mach Numbers From 1.50 to 4.63 . NASA TM X-2365, 1971 . 


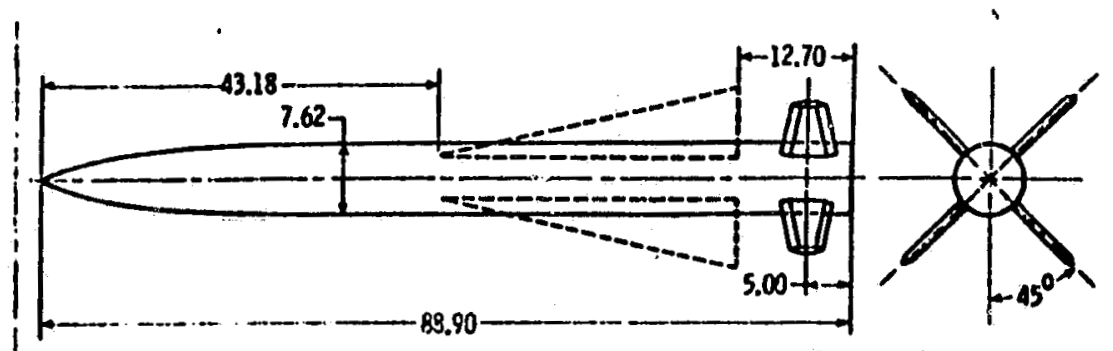

ALL DIMENSIONS ARE IN CENTIMETERS UNLESS OTHERWISE NOTED
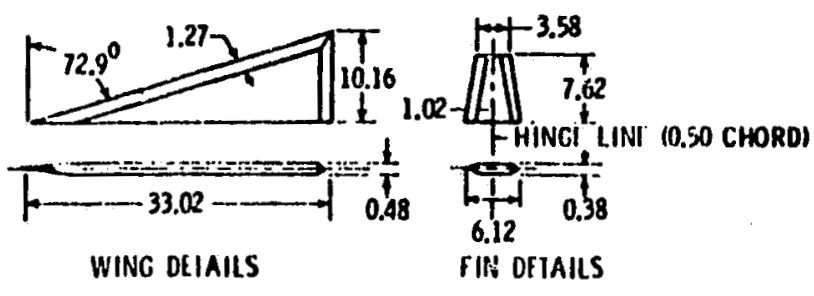

Figure 1. - Details of model. Dimensions in centimeters unless otherwise noted.

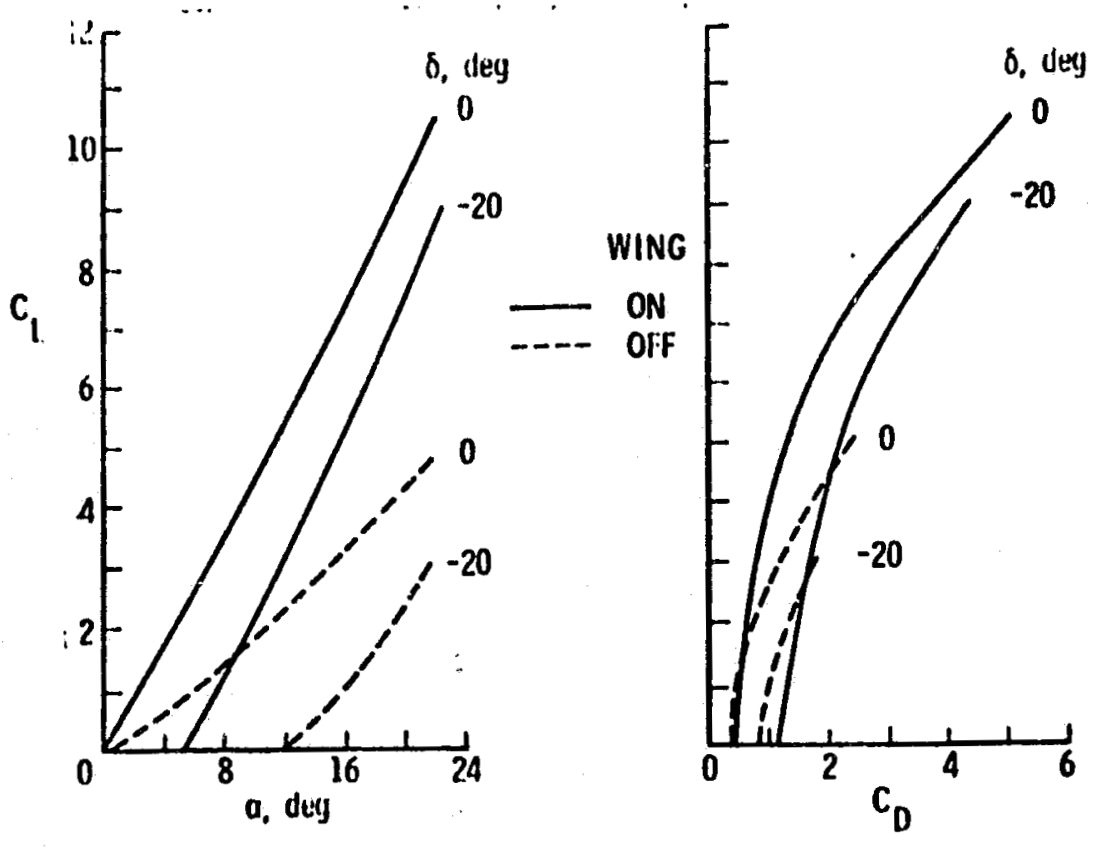

ligure ?. - Lift and drig characteristics, $\phi=45^{\circ}$, $M=1.90$. 


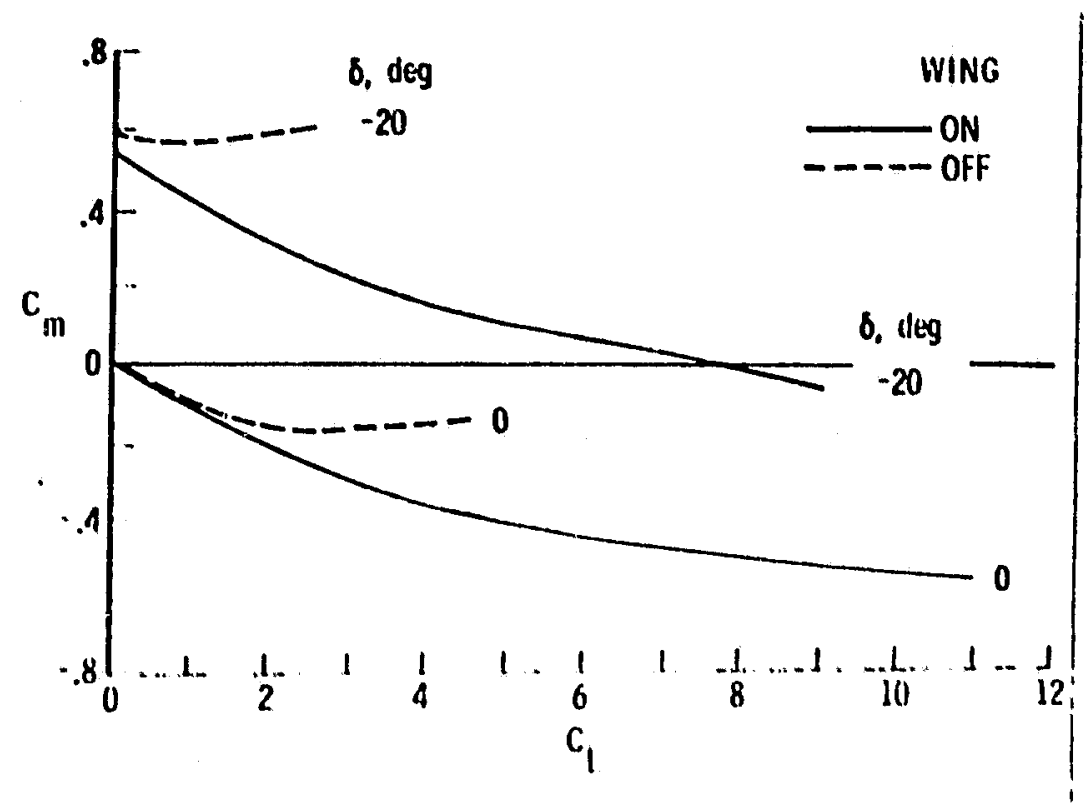

Figure 3. - Stability characteristics, c.g. $=0.60 \mathrm{t}$, $\phi=45^{\circ}, M=1.90$.
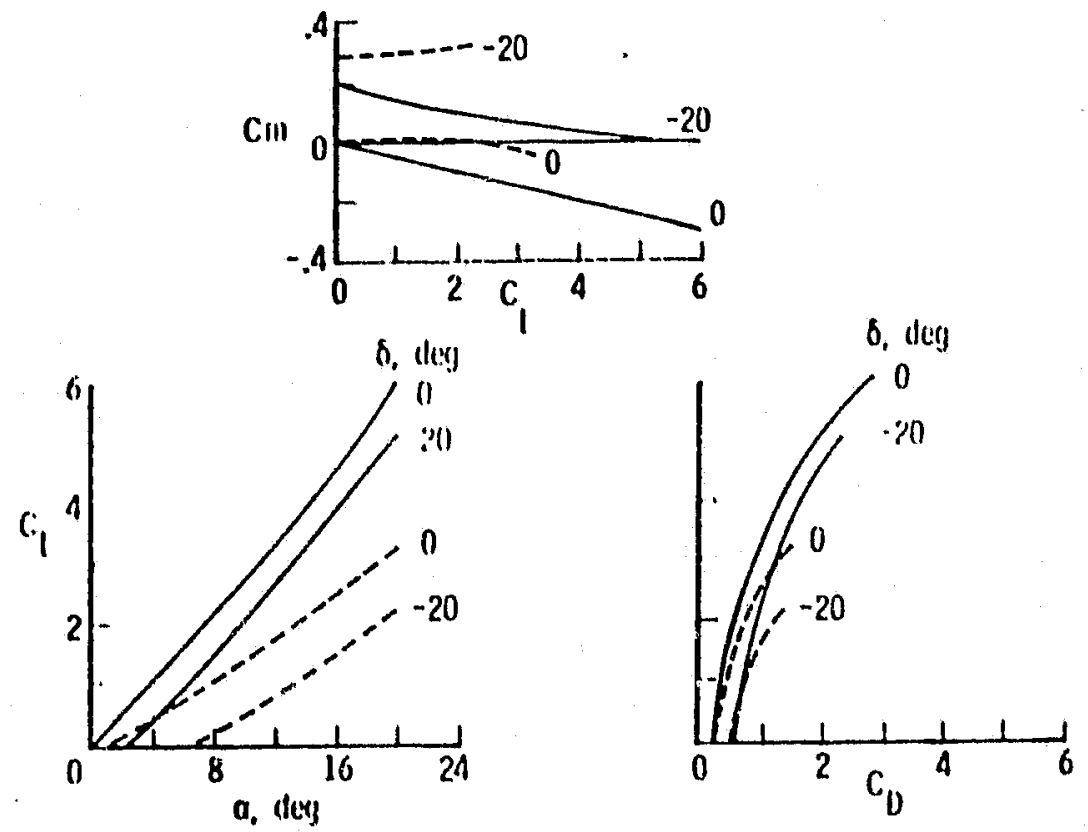

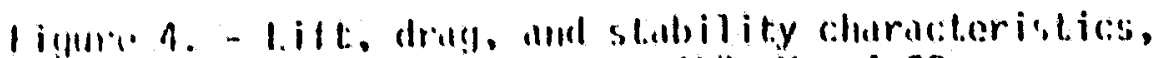
c.g. : $0.601,4.4 !, 0, M=4.63$. 

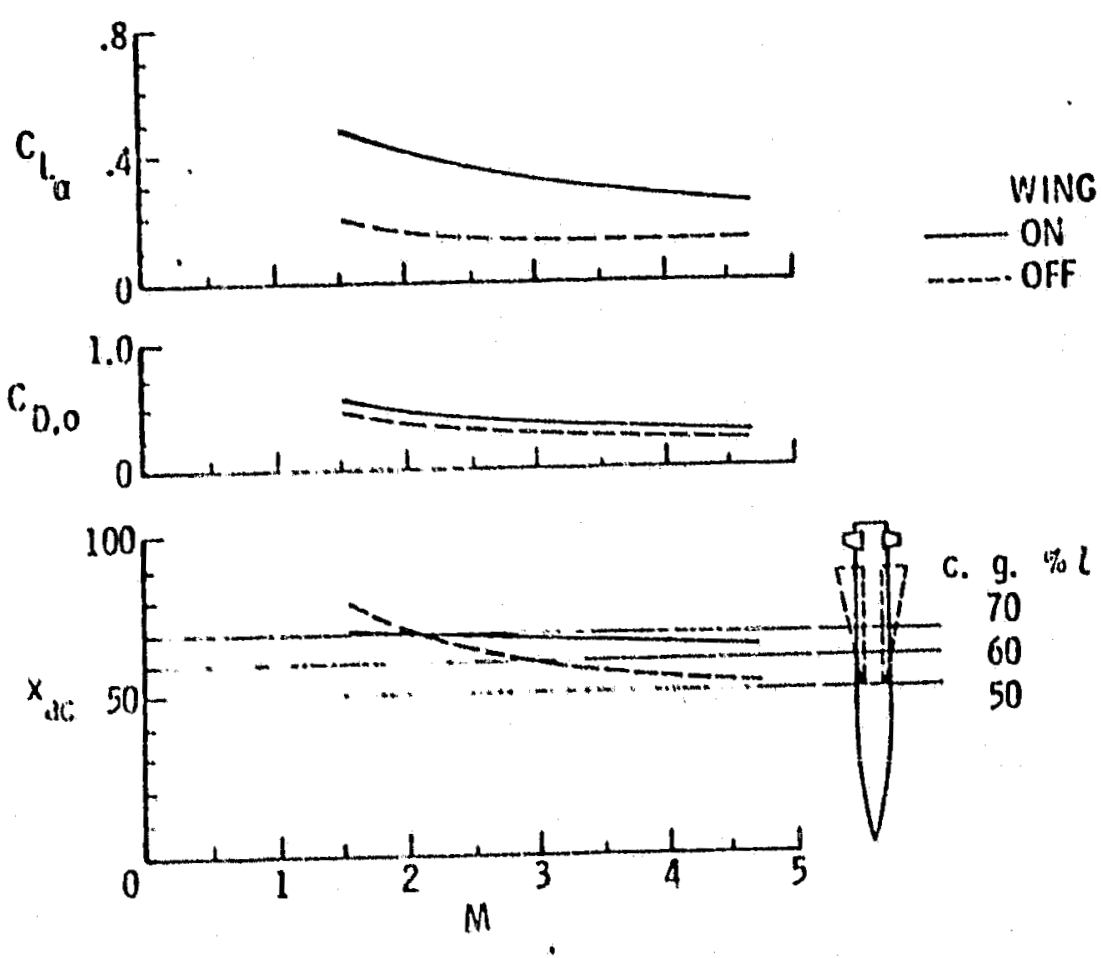

Figure 5. - Longitudinal aerodynanic parameters, $\phi=45^{\circ}$. 


\section{ORIGINAL DAOE IS \\ OF POOR QUALITY}

8. deg

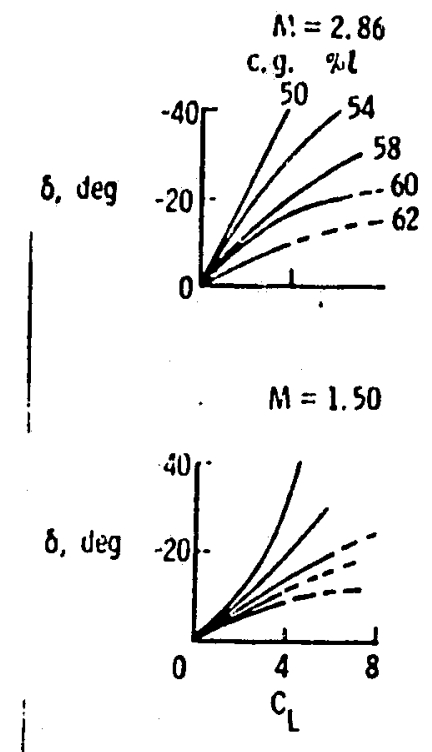

$M=3.95$

$M=4.63$
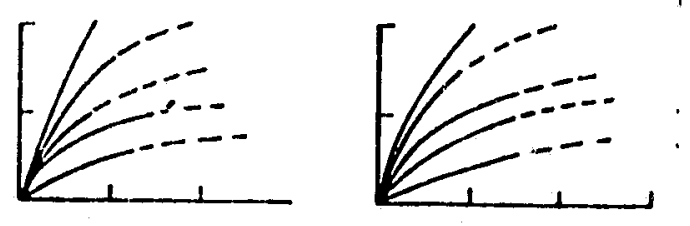

Figure 6. - Tail trim lift effectiveness with wing on, $\phi=45^{\circ}$.
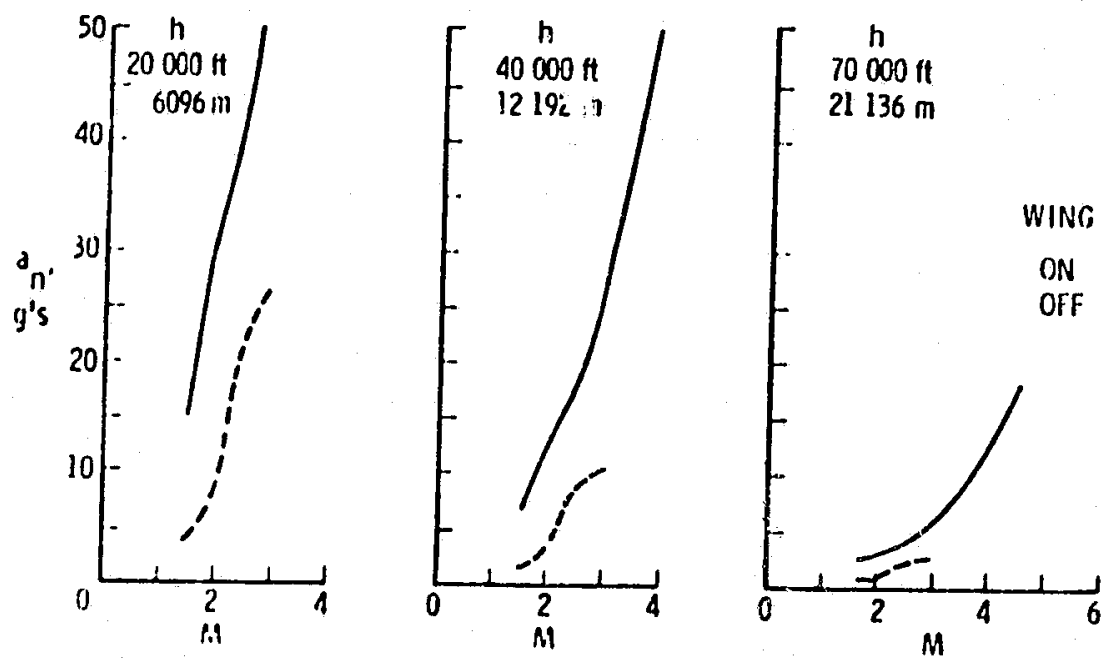

Iigure 7. - Instantaneous normal acceleration, 4 $\because 4 !, 0, W / A=3 ! .9 \mathrm{kN} / \mathrm{m}^{2}$,

C.g. $=0.60 \mathrm{z}$. 